\title{
Oscillation for second-order half-linear delay damped dynamic equations on time scales
}

Jimeng $\mathrm{Li}^{1}$ and Jiashan Yang ${ }^{2 *}$

"Correspondence: syxyyjs@163.com

${ }^{2}$ School of Data Science and

Software Engineering, Wuzhou

University, Wuzhou, P.R. China

Full list of author information is

available at the end of the article

\section{Abstract}

We investigate oscillation of second-order half-linear variable delay damped dynamic equations

$$
\left[a(t)\left|x^{\Delta}(t)\right|^{\lambda-1} x^{\Delta}(t)\right]^{\Delta}+b(t)\left|x^{\Delta}(t)\right|^{\lambda-1} x^{\Delta}(t)+p(t)|x(\delta(t))|^{\lambda-1} x(\delta(t))=0
$$

on a time scale $\mathbb{T}$. By using the generalized Riccati transformation and the inequality technique, we establish some new oscillation criteria for the equations under the condition

$$
\int_{t_{0}}^{\infty}\left[a^{-1}(s) e_{-b / a}\left(s, t_{0}\right)\right]^{1 / \lambda} \Delta s<\infty
$$

These results deal with some cases not covered by existing results in the literature.

MSC: 34K11;34C10;39A10

Keywords: Oscillation; Time scales; Functional dynamic equations; Riccati substitutions; Variable delay

\section{Introduction}

In this paper, we are concerned with the oscillatory behavior of a second-order half-linear damped dynamic equation

$$
\left[a(t)\left|x^{\Delta}(t)\right|^{\lambda-1} x^{\Delta}(t)\right]^{\Delta}+b(t)\left|x^{\Delta}(t)\right|^{\lambda-1} x^{\Delta}(t)+p(t)|x(\delta(t))|^{\lambda-1} x(\delta(t))=0, \quad t \in \mathbb{T},
$$

where $t \geq t_{0}, t_{0}>0$, and $\lambda>0$ are constants. For completeness, we recall the following concepts related to the notion of time scales. A time scale $\mathbb{T}$ is an arbitrary nonempty closed subset of the real numbers $\mathbb{R}$. On the time scale $\mathbb{T}$ we define the forward and the backward jump operators by

$$
\sigma(t)=\inf \{s \in \mathbb{T}: s>t\} \quad \text { and } \quad \rho(t)=\sup \{s \in \mathbb{T}: s<t\}
$$

A point $t \in \mathbb{T}$ is said to be left-dense if $\rho(t)=t$, right-dense if $\sigma(t)=t$, left-scattered if $\rho(t)<t$, and right-scattered if $\sigma(t)>t$. The graininess $\mu$ of the time scale is defined by $\mu(t)=\sigma(t)-t$. For a function $f: \mathbb{T} \rightarrow \mathbb{R}$, the (delta) derivative is defined by $f^{\Delta}(t)=\frac{f(\sigma(t))-f(t)}{\sigma(t)-t}$

(c) The Author(s) 2019. This article is distributed under the terms of the Creative Commons Attribution 4.0 International License (http://creativecommons.org/licenses/by/4.0/), which permits unrestricted use, distribution, and reproduction in any medium, provided you give appropriate credit to the original author(s) and the source, provide a link to the Creative Commons license, and indicate if changes were made. 
if $f$ is continuous at $t$ and $t$ is right-scattered. If $t$ is right-dense, then the derivative is defined by $f^{\Delta}(t)=\lim _{s \rightarrow t^{+}} \frac{f(t)-f(s)}{t-s}$, provided this limit exists. A function $f: \mathbb{T} \rightarrow \mathbb{R}$ is said to be rd-continuous if it is continuous at each right-dense point and if there exists a finite left limit at all left-dense points. The set of rd-continuous functions $f: \mathbb{T} \rightarrow \mathbb{R}$ is denoted by $C_{r d}(\mathbb{T}, \mathbb{R})$. The derivative $f^{\Delta}$ of $f$ and the $\operatorname{shift} f^{\sigma}$ of $f$ are related by the formula $f^{\sigma}=f+\mu f^{\Delta}$ where $f^{\sigma}=f \circ \sigma$.

Throughout, we assume the following hypotheses:

$\left(\mathrm{H}_{1}\right): \mathbb{T}$ is an arbitrary time scale (i.e., a nonempty closed subset of the real numbers $\mathbb{R}$ ) which is unbounded above (i.e., $\sup \mathbb{T}=\infty$ ), and $t_{0} \in \mathbb{T}$ with $t_{0}>0$, we define a time scale interval of the form $\left[t_{0}, \infty\right)_{\mathbb{T}}$ by $\left[t_{0}, \infty\right)_{\mathbb{T}}=\left[t_{0}, \infty\right) \cap \mathbb{T}$.

$\left(\mathrm{H}_{2}\right)$ : the delay function $\delta: \mathbb{T} \rightarrow \mathbb{T}$ is strictly increasing and differentiable, and $\delta(t) \leq$ $t, \lim _{t \rightarrow \infty} \delta(t)=\infty, \delta(\mathbb{T})=\mathbb{T}$.

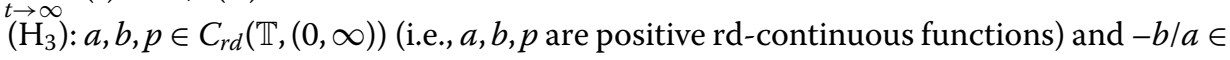
$\Re^{+}$.

By a solution of (1.1) we mean a nontrivial real-valued function $x \in C_{r d}\left(\left[T_{x}, \infty\right)_{\mathbb{T}}, \mathbb{R}\right)$, where $T_{x} \in\left[t_{0}, \infty\right)_{\mathbb{T}}$, which has the property that $a(t)\left|x^{\Delta}(t)\right|^{\lambda-1} x^{\Delta}(t) \in C_{r d}^{1}\left(\left[T_{x}, \infty\right)_{\mathbb{T}}, \mathbb{R}\right)$ and satisfies (1.1) for $t \in\left[T_{x}, \infty\right)_{\mathbb{T}}$. The solutions vanishing in some neighborhood of infinity will be excluded from our consideration. A solution $x$ of (1.1) is said to be oscillatory if it is neither eventually positive nor eventually negative; otherwise, it is nonoscillatory. Equation (1.1) is called oscillatory if all its solutions oscillate.

Recently, there has been an increasing interest in studying oscillatory behavior of solutions to various classes of dynamic equations on time scales, we refer the reader to [1-23] and the references cited therein. In particular, oscillation of dynamic equations with damping attracted significant attention of researchers due to the fact that such equations arise in many real life problems, see [1-5, 9, 12, 13, 16, 17, 20, 22]. For instance, Zhang et al. [1-3, 5] considered the second-order damped dynamic equation (1.1) under the conditions

$$
\int_{t_{0}}^{\infty}\left[a^{-1}(s) e_{-b / a}\left(s, t_{0}\right)\right]^{1 / \lambda} \Delta s=\infty
$$

and

$$
\int_{t_{0}}^{\infty}\left[a^{-1}(s) e_{-b / a}\left(s, t_{0}\right)\right]^{1 / \lambda} \Delta s<\infty,
$$

respectively, and established many sufficient conditions for oscillation of (1.1). The main result is as follows.

Theorem 1.1 (see [1], Theorem 4.1) Assume $\left(\mathrm{H}_{1}\right)-\left(\mathrm{H}_{3}\right)$ and (1.2). If there exists a positive and differentiable function $\varphi: \mathbb{T} \rightarrow \mathbb{R}$ such that

$$
\limsup _{t \rightarrow \infty} \int_{t_{0}}^{t} \varphi(s)\left[p(s)-\frac{a(\delta(s))}{(\lambda+1)^{\lambda+1}\left(\delta^{\Delta}(s)\right)^{\lambda}}\left|\frac{\varphi^{\Delta}(s)}{\varphi(s)}-\frac{b(s)}{a(s)}\right|^{\lambda+1}\right] \Delta s=\infty,
$$

then Eq. (1.1) is oscillatory on $\left[t_{0}, \infty\right)_{\mathbb{T}}$.

From this Theorem 1.1 and its proof, one can obtain various types of Kamenev-type oscillation criteria (such as Theorem 4.2 in [1], etc.) and different classes of Philos-type oscillation criteria for Eq. (1.1) under condition (1.2) (see [2, 3, 5]). 
Theorem 1.2 (see [1], Theorem 4.3) Assume $\left(\mathrm{H}_{1}\right)-\left(\mathrm{H}_{3}\right),(1.3)$, and (1.4). If

$$
\int_{t_{0}}^{\infty}\left[\frac{1}{a(t)} \int_{t_{0}}^{t} e_{-b / a}(t, \sigma(s)) p(s) \Delta s\right]^{1 / \lambda} \Delta t=\infty
$$

then every solution $x(t)$ of Eq. (1.1) is either oscillatory or satisfies $\lim _{t \rightarrow \infty} x(t)=0$.

Obviously, under the case (1.3), Theorem 1.2 (the other results are the same, for example, Theorem 4.3 and Theorem 4.4 in [2], Theorem 3.5 in [9], etc.) cannot ensure that the solution $x(t)$ of Eq. (1.1) is oscillatory. We can see that, in applications, it is inconvenient that every solution $x(t)$ of Eq. (1.1) oscillates or converges to zero, since we do not know under what conditions separately the solutions oscillate or converge to zero.

On the above basis, in [3] and [5], the authors discussed oscillatory criteria of Eq. (1.1), got the result that every solution of Eq. (1.1) oscillates, and improved the results in [1, 2, 9]. One of the results they provided is as follows.

Theorem 1.3 (see [5], Theorem 4.3) Assume $\left(\mathrm{H}_{1}\right)-\left(\mathrm{H}_{3}\right),(1.3)$, and (1.4). If for every $t_{1} \in$ $\left[t_{0}, \infty\right)_{\mathbb{T}}$,

$$
\int_{t_{1}}^{\infty}\left[\frac{1}{a(t)} \int_{t_{1}}^{t} e_{-b / a}(t, \sigma(s)) \theta^{\lambda}(s) p(s) \Delta s\right]^{1 / \lambda} \Delta t=\infty
$$

where $\theta(t)=\int_{t}^{\infty} a^{-1 / \lambda}(s) \Delta s$, then Eq. (1.1) is oscillatory on $\left[t_{0}, \infty\right)_{\mathbb{T}}$.

Using the same method, one can deduce from Theorem 1.3 a great number of oscillation criteria for Eq. (1.1) (see [3, 5]).

On the other hand, it is well known that the Euler differential equation

$$
\left(t^{2} x^{\prime}(t)\right)^{\prime}+p_{0} x(t)=0, \quad t \geq 1,
$$

is oscillatory if $p_{0}>1 / 4$. However, Theorem 1.3 cannot be applied in (1.7) due to $\int_{1}^{\infty} t^{-2} \ln t \mathrm{~d} t<\infty$. One can easily see that the recent results (such as the results in [3, $4,6-8,12-21]$, etc.) cannot be applied in (1.7).

The purpose of this article is to obtain new criteria for the oscillation of (1.1) under condition (1.3) which promote some existing results.

\section{Main results}

Lemma 2.1 ([6]) Assume that $x(t)$ is delta-differentiable and eventually positive or eventually negative, then

$$
\left(x^{\lambda}(t)\right)^{\Delta}=\lambda x^{\Delta}(t) \int_{0}^{1}[h x(\sigma(t))+(1-h) x(t)]^{\lambda-1} \mathrm{~d} h .
$$

Lemma 2.2 (Yang's inequality [17]) Let $A>0, B>0$, and $p>0$ be constants, then $A B \leq$ $\frac{A^{p}}{p}+\frac{B^{q}}{q}$ for $\frac{1}{p}+\frac{1}{q}=1$.

Lemma 2.3 ([21]) Let $\lambda \geq 1$ be a quotient of two odd numbers, then

$$
X^{1+\frac{1}{\lambda}}-(X-Y)^{1+\frac{1}{\lambda}} \leq Y^{\frac{1}{\lambda}}\left[\left(1+\frac{1}{\lambda}\right) X-\frac{1}{\lambda} Y\right], \quad X Y \geq 0 .
$$


Theorem 2.1 Assume $\left(\mathrm{H}_{1}\right)-\left(\mathrm{H}_{3}\right)$, (1.3), and (1.4). If

$$
\limsup _{t \rightarrow \infty} \int_{t_{0}}^{t}\left[p(s) \theta^{\lambda}(\sigma(s))-\frac{a(s) \theta^{\lambda(\lambda+1)}(s)}{(\lambda+1)^{\lambda+1} \theta^{\lambda^{2}}(\sigma(s))}\left(\frac{\bar{\theta}(s)}{\theta^{\lambda}(s)}+\frac{b(s)}{a(s)}\right)^{\lambda+1}\right] \Delta s=\infty,
$$

where

$$
\begin{aligned}
& \theta(t)=\int_{t}^{\infty}\left[a^{-1}(s) e_{-b / a}\left(s, t_{0}\right)\right]^{1 / \lambda} \Delta s, \\
& \bar{\theta}(t)= \begin{cases}\lambda \theta^{\lambda-1}(t)\left[a^{-1}(t) e_{-b / a}\left(t, t_{0}\right)\right]^{1 / \lambda}, & \lambda \geq 1, \\
\lambda \theta^{\lambda-1}(\sigma(t))\left[a^{-1}(t) e_{-b / a}\left(t, t_{0}\right)\right]^{\frac{1}{\lambda}}, & 0<\lambda<1,\end{cases}
\end{aligned}
$$

then Eq. (1.1) is oscillatory on $\left[t_{0}, \infty\right)_{\mathbb{T}}$.

Proof Let $x(t)$ be a nonoscillatory solution of Eq. (1.1). Without loss of generality, we may assume that there exists $t_{1} \in\left[t_{0}, \infty\right)_{\mathbb{T}}$ such that $x(t)>0, x(\delta(t))>0\left(t \in\left[t_{1}, \infty\right)_{\mathbb{T}}\right)$. Then, from (1.1), we have for $t \in\left[t_{1}, \infty\right)_{\mathbb{T}}$

$$
\left[a(t)\left|x^{\Delta}(t)\right|^{\lambda-1} x^{\Delta}(t)\right]^{\Delta}+b(t)\left|x^{\Delta}(t)\right|^{\lambda-1} x^{\Delta}(t)=-p(t) x^{\lambda}(\delta(t))<0 .
$$

Proceeding as in the proof of Lemma 3.5 in [1], we get that $\frac{a(t)\left|x^{\Delta}(t)\right|^{\lambda-1} x^{\Delta}(t)}{e_{-b / a}\left(t, t_{0}\right)}\left(t \in\left[t_{1}, \infty\right)_{\mathbb{T}}\right)$ is decreasing and $x^{\Delta}(t)$ is either eventually positive or eventually negative. Therefore, we shall distinguish the following two cases:

(I) $x^{\Delta}(t)>0, t \in\left[t_{1}, \infty\right)_{\mathbb{T}} ;$ (II) $x^{\Delta}(t)<0, t \in\left[t_{1}, \infty\right)_{\mathbb{T}}$.

Case (I): $x^{\Delta}(t)>0, t \in\left[t_{1}, \infty\right)_{\mathbb{T}}$. As in the proof of [1], Theorem 4.1, one can obtain a contradiction to (1.4).

$$
\begin{aligned}
& \text { Case (II): } x^{\Delta}(t)<0, t \in\left[t_{1}, \infty\right)_{\mathbb{T}} \text {. Let } \\
& \qquad w(t)=\frac{a(t)\left|x^{\Delta}(t)\right|^{\lambda-1} x^{\Delta}(t)}{|x(t)|^{\lambda-1} x(t)}=\frac{a(t)\left(-x^{\Delta}(t)\right)^{\lambda-1} x^{\Delta}(t)}{x^{\lambda}(t)}, \quad t \in\left[t_{1}, \infty\right)_{\mathbb{T}},
\end{aligned}
$$

then $\left.w(t)<0\left(t \in\left[t_{1}, \infty\right)\right)_{\mathbb{T}}\right)$. Since $\frac{a(t)\left|x^{\Delta}(t)\right|^{\lambda-1} x^{\Delta}(t)}{e_{-b / a}\left(t, t_{0}\right)}=\frac{a(t)\left(-x^{\Delta}(t)\right)^{\lambda-1} x^{\Delta}(t)}{e_{-b / a}\left(t, t_{0}\right)}\left(t \in\left[t_{1}, \infty\right) \mathbb{T}\right)$ is decreasing, and therefore, for all $s \in[t, \infty)_{\mathbb{T}}$, we have

$$
\frac{a(s)\left(-x^{\Delta}(s)\right)^{\lambda-1} x^{\Delta}(s)}{e_{-b / a}\left(s, t_{0}\right)} \leq \frac{a(t)\left(-x^{\Delta}(t)\right)^{\lambda-1} x^{\Delta}(t)}{e_{-b / a}\left(t, t_{0}\right)},
$$

hence,

$$
x^{\Delta}(s) \leq\left(\frac{e_{-b / a}\left(s, t_{0}\right)}{e_{-b / a}\left(t, t_{0}\right)}\right)^{1 / \lambda} \frac{a^{1 / \lambda}(t) x^{\Delta}(t)}{a^{1 / \lambda}(s)} .
$$

It follows that

$$
x(u) \leq x(t)+\frac{a^{1 / \lambda}(t) x^{\Delta}(t)}{\left[e_{-b / a}\left(t, t_{0}\right)\right]^{1 / \lambda}} \int_{t}^{u}\left[a^{-1}(s) e_{-b / a}\left(s, t_{0}\right)\right]^{1 / \lambda} \Delta s .
$$

Let $u \rightarrow \infty$, we find that

$$
x(t)+\frac{a^{1 / \lambda}(t) x^{\Delta}(t)}{\left[e_{-b / a}\left(t, t_{0}\right)\right]^{1 / \lambda}} \theta(t) \geq 0 .
$$


In view of $0<e_{-b / a}\left(t, t_{0}\right) \leq 1$ and $x^{\Delta}(t)<0$, we see that $x(t)+a^{1 / \lambda}(t) x^{\Delta}(t) \theta(t) \geq 0$, it follows

$$
-1 \leq \frac{a^{1 / \lambda}(t) x^{\Delta}(t)}{x(t)} \theta(t) \leq 0
$$

By virtue of (2.3) and (2.6), we conclude that

$$
-1 \leq w(t) \theta^{\lambda}(t) \leq 0
$$

By Lemma 2.1 and $x^{\Delta}(t)<0$, it is not difficult to find that

$$
\left\{\begin{array}{l}
\left(x^{\lambda}(t)\right)^{\Delta} \leq \lambda x^{\lambda-1}(\sigma(t)) x^{\Delta}(t), \quad \lambda \geq 1, \\
\left(x^{\lambda}(t)\right)^{\Delta} \leq \lambda x^{\lambda-1}(t) x^{\Delta}(t), \quad 0<\lambda<1 .
\end{array}\right.
$$

If $0<\lambda<1$, in view of (2.8), (2.2), and $x^{\Delta}(t)<0$, from (2.3), we then get

$$
\begin{aligned}
w^{\Delta}(t) & =\frac{\left[a(t)\left|x^{\Delta}(t)\right|^{\lambda-1} x^{\Delta}(t)\right]^{\Delta}}{x^{\lambda}(\sigma(t))}-\frac{a(t)\left(-x^{\Delta}(t)\right)^{\lambda-1} x^{\Delta}(t)\left(x^{\lambda}(t)\right)^{\Delta}}{x^{\lambda}(t) x^{\lambda}(\sigma(t))} \\
& \leq-\frac{p(t) x^{\lambda}(\delta(t))+b(t)\left|x^{\Delta}(t)\right|^{\lambda-1} x^{\Delta}(t)}{x^{\lambda}(\sigma(t))}-\frac{a(t)\left(-x^{\Delta}(t)\right)^{\lambda-1} x^{\Delta}(t) \lambda x^{\lambda-1}(t) x^{\Delta}(t)}{x^{\lambda}(t) x^{\lambda}(\sigma(t))} \\
& \leq-p(t)-\frac{b(t) x^{\lambda}(t)}{a(t) x^{\lambda}(\sigma(t))} \frac{a(t)\left|x^{\Delta}(t)\right|^{\lambda-1} x^{\Delta}(t)}{x^{\lambda}(t)}-\frac{\lambda a(t)\left(-x^{\Delta}(t)\right)^{\lambda-1}\left(x^{\Delta}(t)\right)^{2}}{x^{\lambda+1}(t)} \\
& =-p(t)+\frac{b(t) x^{\lambda}(t)}{a(t) x^{\lambda}(\sigma(t))}(-w(t))-\frac{\lambda}{a^{1 / \lambda}(t)}(-w(t))^{\frac{\lambda+1}{\lambda}} .
\end{aligned}
$$

If $\lambda \geq 1$, in view of (2.8) and $x^{\Delta}(t)<0$, similarly, we can obtain (2.9).

Using $\theta^{\Delta}(t)=-\left[a^{-1}(t) e_{-b / a}\left(t, t_{0}\right)\right]^{1 / \lambda}$ and (2.5), we have

$$
\begin{aligned}
\left(\frac{x(t)}{\theta(t)}\right)^{\Delta} & =\frac{x^{\Delta}(t) \theta(t)+x(t)\left[a^{-1}(t) e_{-b / a}\left(t, t_{0}\right)\right]^{1 / \lambda}}{\theta(t) \theta(\sigma(t))} \\
& \geq \frac{x^{\Delta}(t) \theta(t)-\frac{a^{1 / \lambda}(t) x^{\Delta}(t)}{\left[e_{-b / a}\left(t, t_{0}\right)\right]^{1 / \lambda}} \theta(t)\left[a^{-1}(t) e_{-b / a}\left(t, t_{0}\right)\right]^{1 / \lambda}}{\theta(t) \theta(\sigma(t))}=0 .
\end{aligned}
$$

Consequently, the function $\frac{x(t)}{\theta(t)}$ is non-decreasing, and therefore,

$$
\frac{x(t)}{x(\sigma(t))} \leq \frac{\theta(t)}{\theta(\sigma(t))}
$$

Substituting (2.10) into (2.9), we obtain

$$
w^{\Delta}(t) \leq-p(t)+\frac{b(t) \theta^{\lambda}(t)}{a(t) \theta^{\lambda}(\sigma(t))}(-w(t))-\frac{\lambda}{a^{1 / \lambda}(t)}(-w(t))^{\frac{\lambda+1}{\lambda}} .
$$

By Lemma 2.1 and $\theta^{\Delta}(t)<0$, it is easy to show that

$$
\left[\theta^{\lambda}(t)\right]^{\Delta} \geq \begin{cases}-\lambda \theta^{\lambda-1}(t)\left[a^{-1}(t) e_{-b / a}\left(t, t_{0}\right)\right]^{1 / \lambda}, & \lambda \geq 1, \\ -\lambda \theta^{\lambda-1}(\sigma(t))\left[a^{-1}(t) e_{-b / a}\left(t, t_{0}\right)\right]^{\frac{1}{\lambda}}, & 0<\lambda<1 .\end{cases}
$$


That is,

$$
\left[\theta^{\lambda}(t)\right]^{\Delta} \geq-\bar{\theta}(t)
$$

Multiplying (2.11) by $\theta^{\lambda}(\sigma(t))$ and then integrating from $t_{1}$ to $t$, and using the integration by parts formula on time scales, (2.13), and Lemma 2.2, we are led to

$$
\begin{aligned}
\int_{t_{1}}^{t} p(s) & \theta^{\lambda}(\sigma(s)) \Delta s \\
\leq & -\int_{t_{1}}^{t} \theta^{\lambda}(\sigma(s)) w^{\Delta}(s) \Delta s \\
& +\int_{t_{1}}^{t} \theta^{\lambda}(\sigma(s))\left[\frac{b(s) \theta^{\lambda}(s)(-w(s))}{a(s) \theta^{\lambda}(\sigma(s))}-\frac{\lambda(-w(s))^{\frac{\lambda+1}{\lambda}}}{a^{1 / \lambda}(s)}\right] \Delta s \\
= & \theta^{\lambda}\left(t_{1}\right) w\left(t_{1}\right)-\theta^{\lambda}(t) w(t)+\int_{t_{1}}^{t}\left[\theta^{\lambda}(s)\right]^{\Delta} w(s) \Delta s \\
& +\int_{t_{1}}^{t} \theta^{\lambda}(\sigma(s))\left[\frac{b(s) \theta^{\lambda}(s)(-w(s))}{a(s) \theta^{\lambda}(\sigma(s))}-\frac{\lambda(-w(s))^{\frac{\lambda+1}{\lambda}}}{a^{1 / \lambda}(s)}\right] \Delta s \\
\leq & \theta^{\lambda}\left(t_{1}\right) w\left(t_{1}\right)-\theta^{\lambda}(t) w(t) \\
& +\int_{t_{1}}^{t}\left[\left(\bar{\theta}(s)+\frac{b(s) \theta^{\lambda}(s)}{a(s)}\right)(-w(s))-\frac{\lambda \theta^{\lambda}(\sigma(s))}{a^{1 / \lambda}(s)}(-w(s))^{\frac{\lambda+1}{\lambda}}\right] \Delta s .
\end{aligned}
$$

Now let

$$
p=\frac{\lambda+1}{\lambda}, \quad q=\lambda+1
$$

and

$$
A=\frac{(\lambda+1)^{\frac{\lambda}{\lambda+1}} \theta^{\frac{\lambda^{2}}{\lambda+1}}(\sigma(s))}{a^{\frac{1}{\lambda+1}}(s)}(-w(s)), \quad B=\frac{a^{\frac{1}{\lambda+1}}(s) \theta^{\lambda}(s)}{(\lambda+1)^{\frac{\lambda}{\lambda+1}} \theta^{\frac{\lambda^{2}}{\lambda+1}}(\sigma(s))}\left(\frac{\bar{\theta}(s)}{\theta^{\lambda}(s)}+\frac{b(s)}{a(s)}\right) .
$$

Using the inequality (see Lemma 2.2)

$$
A B-\frac{A^{p}}{p} \leq \frac{B^{q}}{q}
$$

we find

$$
\begin{gathered}
\left(\bar{\theta}(s)+\frac{b(s) \theta^{\lambda}(s)}{a(s)}\right)(-w(s))-\frac{\lambda \theta^{\lambda}(\sigma(s))}{a^{1 / \lambda}(s)}(-w(s))^{\frac{\lambda+1}{\lambda}} \\
\leq \frac{a(s) \theta^{\lambda(\lambda+1)}(s)}{(\lambda+1)^{\lambda+1} \theta^{\lambda^{2}}(\sigma(s))}\left(\frac{\bar{\theta}(s)}{\theta^{\lambda}(s)}+\frac{b(s)}{a(s)}\right)^{\lambda+1} .
\end{gathered}
$$

Hence, we obtain

$$
\int_{t_{1}}^{t} p(s) \theta^{\lambda}(\sigma(s)) \Delta s
$$




$$
\leq \theta^{\lambda}\left(t_{1}\right) w\left(t_{1}\right)-\theta^{\lambda}(t) w(t)+\int_{t_{1}}^{t} \frac{a(s) \theta^{\lambda(\lambda+1)}(s)}{(\lambda+1)^{\lambda+1} \theta^{\lambda^{2}}(\sigma(s))}\left(\frac{\bar{\theta}(s)}{\theta^{\lambda}(s)}+\frac{b(s)}{a(s)}\right)^{\lambda+1} \Delta s .
$$

By virtue of (2.7) and (2.14), we conclude that

$$
\int_{t_{1}}^{t}\left[p(s) \theta^{\lambda}(\sigma(s))-\frac{a(s) \theta^{\lambda(\lambda+1)}(s)}{(\lambda+1)^{\lambda+1} \theta^{\lambda^{2}}(\sigma(s))}\left(\frac{\bar{\theta}(s)}{\theta^{\lambda}(s)}+\frac{b(s)}{a(s)}\right)^{\lambda+1}\right] \Delta s \leq \theta^{\lambda}\left(t_{1}\right) w\left(t_{1}\right)+1,
$$

taking the limsup as $t \rightarrow \infty$, then we get a contradiction to condition (2.1). The proof is complete.

Theorem 2.2 Assume $\left(\mathrm{H}_{1}\right)-\left(\mathrm{H}_{3}\right),(1.3),(1.4)$, and $\lambda \geq 1$ is a quotient of two odd numbers. Suppose further that there exist two functions $\psi, \xi \in C^{1}(\mathbb{T},(0, \infty))$ with $\xi(t) \geq \frac{1}{\theta^{\lambda}(t) a(t)}$ such that

$$
\begin{aligned}
& \limsup _{t \rightarrow \infty} \int_{t_{0}}^{t} \theta^{\lambda}(\sigma(s))\left[\psi(\sigma(s)) \Phi(s)-\frac{a(s) \psi^{\lambda+1}(s)\left|\Theta(s)-\frac{\lambda\left(e_{-b / a}\left(s, t_{0}\right)\right)^{1 / \lambda}}{\theta(\sigma(s)) a^{1 / \lambda}(s)}\right|^{\lambda+1}}{(\lambda+1)^{\lambda+1} \psi^{\lambda}(\sigma(s))}\right] \Delta s \\
& \quad=\infty
\end{aligned}
$$

where the function $\theta(t)$ is defined as in Theorem 2.1,

$$
\begin{aligned}
& \Phi(t)=p(t)-[\xi(t) a(t)]^{\Delta}-\frac{b(t)}{a(t) \theta^{\lambda}(\sigma(t))}+a(t) \xi^{\frac{\lambda+1}{\lambda}}(t), \\
& \Theta(t)=\frac{\psi^{\Delta}(t)}{\psi(t)}+\frac{(\lambda+1) \psi(\sigma(t)) \xi^{\frac{1}{\lambda}}(t)}{\psi(t)},
\end{aligned}
$$

then Eq. (1.1) is oscillatory on $\left[t_{0}, \infty\right)_{\mathbb{T}}$.

Proof Let $x(t)$ be a nonoscillatory solution of Eq. (1.1), say, $x(t)>0$ and $x(\delta(t))>0$ for all $t \in\left[t_{1}, \infty\right)_{\mathbb{T}}$ for some $t_{1} \in\left[t_{0}, \infty\right)_{\mathbb{T}}$. Similar to the proof of Theorem 2.1, we consider two cases. Assume first that $x^{\Delta}(t)>0$ for $t \in\left[t_{1}, \infty\right)_{\mathbb{T}}$, by (1.4), this case is not true. Assume now that $x^{\Delta}(t)<0$ for $t \in\left[t_{1}, \infty\right)_{\mathbb{T}}$, we proceed as in the proof of Theorem 2.1 to obtain (2.6) for $t \in\left[t_{1}, \infty\right)_{\mathbb{T}}$. Then, by (2.6), we are led to

$$
x^{\lambda}(t) \geq a(t)\left(-x^{\Delta}(t)\right)^{\lambda} \theta^{\lambda}(t)
$$

which yields

$$
\left(x^{\Delta}(t)\right)^{\lambda}+\frac{x^{\lambda}(t)}{a(t) \theta^{\lambda}(t)} \geq 0, \quad t \in\left[t_{1}, \infty\right)_{\mathbb{T}}
$$

We introduce a generalized Riccati transformation

$$
\begin{aligned}
v(t) & =\psi(t)\left[\frac{a(t)\left|x^{\Delta}(t)\right|^{\lambda-1} x^{\Delta}(t)}{x^{\lambda}(t)}+\xi(t) a(t)\right] \\
& =\psi(t)\left[\frac{a(t)\left(x^{\Delta}(t)\right)^{\lambda}}{x^{\lambda}(t)}+\xi(t) a(t)\right], \quad t \in\left[t_{1},+\infty\right)_{\mathbb{T}} .
\end{aligned}
$$


Then, it is not hard to see that $v(t) \geq 0\left(t \in\left[t_{1},+\infty\right)_{\mathbb{T}}\right)$ due to $(2.16)$ and the definition of $\xi(t)$. In view of (2.2), the first formula of (2.8), (2.16), and $x^{\Delta}(t)<0$, respectively, it follows from (2.17) that

$$
\begin{aligned}
v^{\Delta}(t)= & \psi^{\Delta}(t)\left[\frac{a(t)\left(x^{\Delta}(t)\right)^{\lambda}}{x^{\lambda}(t)}+\xi(t) a(t)\right]+\psi(\sigma(t))\left[\frac{a(t)\left(x^{\Delta}(t)\right)^{\lambda}}{x^{\lambda}(t)}+\xi(t) a(t)\right]^{\Delta} \\
= & \frac{\psi^{\Delta}(t)}{\psi(t)} v(t)+\psi(\sigma(t))\left\{[\xi(t) a(t)]^{\Delta}+\frac{\left[a(t)\left(x^{\Delta}(t)\right)^{\lambda}\right]^{\Delta}}{x^{\lambda}(\sigma(t))}-\frac{a(t)\left(x^{\Delta}(t)\right)^{\lambda}\left[x^{\lambda}(t)\right]^{\Delta}}{x^{\lambda}(t) x^{\lambda}(\sigma(t))}\right\} \\
\leq & \frac{\psi^{\Delta}(t)}{\psi(t)} v(t)+\psi(\sigma(t))\left\{[\xi(t) a(t)]^{\Delta}-\frac{p(t) x^{\lambda}(\delta(t))+b(t)\left(x^{\Delta}(t)\right)^{\lambda}}{x^{\lambda}(\sigma(t))}\right. \\
& \left.-\frac{\lambda a(t)\left(x^{\Delta}(t)\right)^{\lambda} x^{\Delta}(t)}{x^{\lambda}(t) x(\sigma(t))}\right\} \\
\leq & \frac{\psi^{\Delta}(t)}{\psi(t)} v(t)+\psi(\sigma(t))\left\{[\xi(t) a(t)]^{\Delta}-p(t)-\frac{b(t)\left(x^{\Delta}(t)\right)^{\lambda}}{x^{\lambda}(\sigma(t))}-\frac{\lambda a(t)\left(x^{\Delta}(t)\right)^{\lambda+1}}{x^{\lambda+1}(t)}\right\} \\
\leq & \frac{\psi^{\Delta}(t)}{\psi(t)} v(t)+\psi(\sigma(t))\left\{[\xi(t) a(t)]^{\Delta}-p(t)+\frac{b(t) x^{\lambda}(t)}{x^{\lambda}(\sigma(t)) a(t) \theta^{\lambda}(t)}\right. \\
& \left.-\frac{\lambda}{a^{\frac{1}{\lambda}}(t)}\left(\frac{v(t)}{\psi(t)}-\xi(t) a(t)\right)^{\frac{\lambda+1}{\lambda}}\right\} .
\end{aligned}
$$

By Lemma 2.3,

$$
(X-Y)^{1+\frac{1}{\lambda}} \geq X^{1+\frac{1}{\lambda}}+\frac{1}{\lambda} Y^{1+\frac{1}{\lambda}}-\left(1+\frac{1}{\lambda}\right) X Y^{\frac{1}{\lambda}},
$$

where $\lambda \geq 1$ is a quotient of two odd numbers, $X Y \geq 0$. Let $X=\frac{v(t)}{\psi(t)}, Y=\xi(t) a(t)$, then we have

$$
\left(\frac{v(t)}{\psi(t)}-\xi(t) a(t)\right)^{\frac{\lambda+1}{\lambda}} \geq \frac{v^{\frac{\lambda+1}{\lambda}}(t)}{\psi^{\frac{\lambda+1}{\lambda}}(t)}+\frac{1}{\lambda}[\xi(t) a(t)]^{\frac{\lambda+1}{\lambda}}-\left(1+\frac{1}{\lambda}\right) \frac{[\xi(t) a(t)]^{\frac{1}{\lambda}}}{\psi(t)} v(t) .
$$

In view of (2.10), (2.19), the definition of $\Phi(t)$ and $\Theta(t)$, it follows from (2.18) that

$$
\begin{aligned}
v^{\Delta}(t) \leq & \frac{\psi^{\Delta}(t)}{\psi(t)} v(t)+\psi(\sigma(t))\left\{[\xi(t) a(t)]^{\Delta}-p(t)+\frac{b(t)}{\theta^{\lambda}(t) a(t)} \frac{\theta^{\lambda}(t)}{\theta^{\lambda}(\sigma(t))}\right. \\
& \left.-\frac{\lambda}{a^{\frac{1}{\lambda}}(t) \psi{ }^{\frac{\lambda+1}{\lambda}}(t)} v^{\frac{\lambda+1}{\lambda}}(t)-a(t) \xi^{\frac{\lambda+1}{\lambda}}(t)+\frac{(\lambda+1) \xi^{\frac{1}{\lambda}}(t)}{\psi(t)} v(t)\right\} \\
= & -\psi(\sigma(t)) \Phi(t)+\Theta(t) v(t)-\frac{\lambda \psi(\sigma(t))}{a^{\frac{1}{\lambda}}(t) \psi^{\frac{\lambda+1}{\lambda}}(t)} v^{\frac{\lambda+1}{\lambda}}(t) .
\end{aligned}
$$

Using the integration by parts formula on time scales, (2.13), and Lemma 2.2, it follows now from (2.20) that

$$
\begin{aligned}
& \int_{t_{1}}^{t} \theta^{\lambda}(\sigma(s)) \psi(\sigma(s)) \Phi(s) \Delta s \\
& \quad \leq-\int_{t_{1}}^{t} \theta^{\lambda}(\sigma(s)) v^{\Delta}(s) \Delta s+\int_{t_{1}}^{t} \theta^{\lambda}(\sigma(s))\left[\Theta(s) v(s)-\frac{\lambda \psi(\sigma(s)) v^{\frac{\lambda+1}{\lambda}}(s)}{a^{\frac{1}{\lambda}}(s) \psi^{\frac{\lambda+1}{\lambda}}(s)}\right] \Delta s
\end{aligned}
$$




$$
\begin{aligned}
= & -\theta^{\lambda}(t) v(t)+\theta^{\lambda}\left(t_{1}\right) v\left(t_{1}\right)+\int_{t_{1}}^{t}\left[\theta^{\lambda}(s)\right]^{\Delta} v(s) \Delta s \\
& +\int_{t_{1}}^{t} \theta^{\lambda}(\sigma(s))\left[\Theta(s) v(s)-\frac{\lambda \psi(\sigma(s)) v^{\frac{\lambda+1}{\lambda}}(s)}{a^{\frac{1}{\lambda}}(s) \psi^{\frac{\lambda+1}{\lambda}}(s)}\right] \Delta s \\
\leq & \theta^{\lambda}\left(t_{1}\right) v\left(t_{1}\right)+\int_{t_{1}}^{t} \theta^{\lambda}(\sigma(s))\left[\left(\Theta(s)-\frac{\lambda\left(e_{-b / a}\left(s, t_{0}\right)\right)^{1 / \lambda}}{\theta(\sigma(s)) a^{1 / \lambda}(s)}\right) v(s)\right. \\
& \left.-\frac{\lambda \psi(\sigma(s)) v^{\frac{\lambda+1}{\lambda}}(s)}{a^{\frac{1}{\lambda}}(s) \psi^{\frac{\lambda+1}{\lambda}}(s)}\right] \Delta s .
\end{aligned}
$$

Now let

$$
p=\frac{\lambda+1}{\lambda}, \quad q=\lambda+1
$$

and

$$
A=\frac{(\lambda+1)^{\frac{\lambda}{\lambda+1}} \psi^{\frac{\lambda}{\lambda+1}}(\sigma(s))}{a^{\frac{1}{\lambda+1}}(s) \psi(s)} v(s), \quad B=\frac{a^{\frac{1}{\lambda+1}}(s) \psi(s)}{(\lambda+1)^{\frac{\lambda}{\lambda+1}} \psi^{\frac{\lambda}{\lambda+1}}(\sigma(s))}\left|\Theta(s)-\frac{\lambda\left(e_{-b / a}\left(s, t_{0}\right)\right)^{1 / \lambda}}{\theta(\sigma(s)) a^{1 / \lambda}(s)}\right| .
$$

Using the inequality (see Lemma 2.2)

$$
A B-\frac{A^{p}}{p} \leq \frac{B^{q}}{q}
$$

we have

$$
\begin{aligned}
& \left(\Theta(s)-\frac{\lambda\left(e_{-b / a}\left(s, t_{0}\right)\right)^{\frac{1}{\lambda}}}{\theta(\sigma(s)) a^{\frac{1}{\lambda}}(s)}\right) v(s)-\frac{\lambda \psi(\sigma(s)) v^{\frac{\lambda+1}{\lambda}}(s)}{a^{\frac{1}{\lambda}}(s) \psi^{\frac{\lambda+1}{\lambda}}(s)} \\
& \leq \frac{a(s) \psi^{\lambda+1}(s)}{(\lambda+1)^{\lambda+1} \psi^{\lambda}(\sigma(s))}\left|\Theta(s)-\frac{\lambda\left(e_{-b / a}\left(s, t_{0}\right)\right)^{\frac{1}{\lambda}}}{\theta(\sigma(s)) a^{\frac{1}{\lambda}}(s)}\right|^{\lambda+1} .
\end{aligned}
$$

By virtue of (2.21) and the above inequality, we conclude that

$$
\begin{aligned}
& \int_{t_{1}}^{t} \theta^{\lambda}(\sigma(s)) \psi(\sigma(s)) \Phi(s) \Delta s \\
& \quad \leq \theta^{\lambda}\left(t_{1}\right) v\left(t_{1}\right)+\int_{t_{1}}^{t} \theta^{\lambda}(\sigma(s))\left[\frac{a(s) \psi^{\lambda+1}(s)}{(\lambda+1)^{\lambda+1} \psi^{\lambda}(\sigma(s))}\left|\Theta(s)-\frac{\lambda\left(e_{-b / a}\left(s, t_{0}\right)\right)^{1 / \lambda}}{\theta(\sigma(s)) a^{1 / \lambda}(s)}\right|^{\lambda+1}\right] \Delta s ;
\end{aligned}
$$

consequently,

$$
\begin{aligned}
& \int_{t_{1}}^{t} \theta^{\lambda}(\sigma(s))\left[\psi(\sigma(s)) \Phi(s)-\frac{a(s) \psi^{\lambda+1}(s)}{(\lambda+1)^{\lambda+1} \psi^{\lambda}(\sigma(s))}\left|\Theta(s)-\frac{\lambda\left(e_{-b / a}\left(s, t_{0}\right)\right)^{1 / \lambda}}{\theta(\sigma(s)) a^{1 / \lambda}(s)}\right|^{\lambda+1}\right] \Delta s \\
& \quad \leq \theta^{\lambda}\left(t_{1}\right) v\left(t_{1}\right),
\end{aligned}
$$

which leads to a contradiction with (2.15). The proof is complete. 
Example 2.1 Consider the second-order Euler differential equation (1.7), i.e.,

$$
\left(t^{2} x^{\prime}(t)\right)^{\prime}+p_{0} x(t)=0, \quad t \geq 1,
$$

here $p_{0}>0$ is a constant. Let $a(t)=t^{2}, b(t)=0, P(t)=p_{0}, \delta(t)=t, \lambda=1, t_{0}=1$, clearly, conditions $\left(\mathrm{H}_{1}\right)-\left(\mathrm{H}_{3}\right)$ and $(1.3)$ are satisfied. Since $\mathbb{T}=\mathbb{R}$, we see that

$$
\theta(t)=\int_{t}^{+\infty}\left[a^{-1}(s) e_{-b / a}\left(s, t_{0}\right)\right]^{1 / \lambda} \Delta s=\int_{t}^{+\infty} s^{-2} \mathrm{~d} s=\frac{1}{t},
$$

and

$$
\bar{\theta}(t)=\lambda \theta^{\lambda-1}(t)\left[a^{-1}(t) e_{-b / a}\left(t, t_{0}\right)\right]^{1 / \lambda}=t^{-2}, \quad \pi(t)=1, \quad \Psi(t)=p_{0} .
$$

Now, pick $\varphi(t)=1$, then

$$
\limsup _{t \rightarrow \infty} \int_{t_{0}}^{t} \varphi(s)\left[p(s)-\frac{a(\delta(s))}{(\lambda+1)^{\lambda+1}\left(\delta^{\Delta}(s)\right)^{\lambda}}\left|\frac{\varphi^{\Delta}(s)}{\varphi(s)}-\frac{b(s)}{a(s)}\right|^{\lambda+1}\right] \Delta s=\limsup _{t \rightarrow \infty} \int_{1}^{t} p_{0} \mathrm{~d} s=\infty,
$$

and

$$
\begin{aligned}
& \limsup _{t \rightarrow \infty} \int_{t_{0}}^{t}\left[p(s) \theta^{\lambda}(\sigma(s))-\frac{a(s) \theta^{\lambda(\lambda+1)}(s)}{(\lambda+1)^{\lambda+1} \theta^{\lambda^{2}(\sigma(s))}}\left(\frac{\bar{\theta}(s)}{\theta^{\lambda}(s)}+\frac{b(s)}{a(s)}\right)^{\lambda+1}\right] \Delta s \\
& \quad=\left(p_{0}-\frac{1}{4}\right) \limsup _{t \rightarrow \infty} \int_{1}^{t} \frac{1}{s} \mathrm{~d} s=\infty,
\end{aligned}
$$

provided that $p_{0}>1 / 4$. Therefore, by Theorem 2.1, the Euler equation (1.7) is oscillatory when $p_{0}>1 / 4$. This conclusion is sharp.

Remark 2.1 Application of Theorem 1.2 or the corresponding result in [1, 2, 9] implies that the every solution $x(t)$ of the Euler equation (1.7) is either oscillatory or satisfies $\lim _{t \rightarrow \infty} x(t)=0$. The results in $[3,5]$ cannot be applied in (1.7) due to $\int_{1}^{\infty} t^{-2} \ln t \mathrm{~d} t<\infty$. One can easily find that the results in $[4,7,8,12-23]$ cannot be applied in (1.7).

Example 2.2 Consider the second-order dynamic equation

$$
\left[t^{2} x^{\Delta}(t)\right]^{\Delta}+p_{0} \frac{2 t-1}{t} x\left(\frac{t}{2}\right)=0, \quad t \in \mathbb{T}=2^{\mathbf{z}}, t \geq 2,
$$

where $p_{0}>0$ is a constant. Obviously, conditions $\left(\mathrm{H}_{1}\right)-\left(\mathrm{H}_{3}\right)$ and $(1.3)$ are satisfied, and we see that

$$
\theta(t)=\int_{t}^{\infty} s^{-2} \Delta s=\lim _{u \rightarrow \infty} \frac{u^{-1}-t^{-1}}{t^{-1}-1}=\frac{1}{t-1}, \quad \bar{\theta}(t)=\frac{1}{t^{2}} .
$$

Now, pick $\varphi(t)=1$, then we have

$$
\limsup _{t \rightarrow \infty} \int_{t_{0}}^{t} \varphi(s)\left[p(s)-\frac{a(\delta(s))}{(\lambda+1)^{\lambda+1}\left(\delta^{\Delta}(s)\right)^{\lambda}}\left|\frac{\varphi^{\Delta}(s)}{\varphi(s)}-\frac{b(s)}{a(s)}\right|^{\lambda+1}\right] \Delta s
$$




$$
=p_{0} \limsup _{t \rightarrow \infty} \int_{2}^{t} \frac{2 s-1}{s} \Delta s=\infty,
$$

and

$$
\begin{aligned}
& \limsup _{t \rightarrow \infty} \int_{t_{0}}^{t}\left[p(s) \theta^{\lambda}(\sigma(s))-\frac{a(s) \theta^{\lambda(\lambda+1)}(s)}{(\lambda+1)^{\lambda+1} \theta^{\lambda^{2}}(\sigma(s))}\left(\frac{\bar{\theta}(s)}{\theta^{\lambda}(s)}+\frac{b(s)}{a(s)}\right)^{\lambda+1}\right] \Delta s \\
& \quad=\limsup _{t \rightarrow \infty} \int_{2}^{t}\left[\left(p_{0}-\frac{1}{2}\right) \frac{1}{s}+\frac{1}{4 s^{2}}\right] \Delta s=\infty,
\end{aligned}
$$

provided that $p_{0}>1 / 2$. Therefore, by Theorem 2.1 , equation (2.2) is oscillatory when $p_{0}>$ $1 / 2$.

Remark 2.2 One can easily find that the results in [1-5, 7-23] cannot be applied in (2.22).

\section{Acknowledgements}

The authors express their sincere gratitude to the editors and referees for the careful reading of the original manuscript and useful comments that helped to improve the presentation of the results and accentuate important details. This work was supported by NNSF of P.R. China (Grant No. 51765060) and the natural science fund project of Hunan Province (Grant No. 12JJ3008).

\section{Funding}

This research was partially supported by the National Natural Science Foundation of China (Grant No. 51765060).

Research Fund for the natural science fund project of Hunan Province (Grant No. 12JJ3008).

\section{Competing interests}

The authors declare that they have no competing interests.

\section{Authors' contributions}

Both authors contributed equally to this work. They both read and approved the final version of the manuscript.

\section{Author details}

${ }^{1}$ School of Science, Shaoyang University, Shaoyang, P.R. China. ${ }^{2}$ School of Data Science and Software Engineering, Wuzhou University, Wuzhou, P.R. China.

\section{Publisher's Note}

Springer Nature remains neutral with regard to jurisdictional claims in published maps and institutional affiliations.

Received: 27 March 2019 Accepted: 10 May 2019 Published online: 20 June 2019

\section{References}

1. Zhang, Q., Gao, L.: Oscillation criteria for second-order half-linear delay dynamic equations with damping on time scales. Sci. Sin., Math. 40, 673-682 (2010)

2. Zhang, Q., Gao, L., Liu, S.: Oscillation criteria for second-order half-linear delay dynamic equations with damping on time scales. Sci. Sin., Math. 43, 793-806 (2013)

3. Zhang, Q., Gao, L., Liu, S.: Oscillation criteria for second-order half-linear delay dynamic equations with damping on time scales (II). Sci. Sin., Math. 41, 885-896 (2011)

4. Erbe, L., Hassan, T.S., Peterson, A.: Oscillation criteria for nonlinear damped dynamic equations on time scales. Appl. Math. Comput. 203, 343-357 (2008)

5. Zhang, Q.: Oscillation of second-order half-linear delay dynamic equations with damping on time scales. J. Comput. Appl. Math. 235, 1180-1188 (2011)

6. Bohner, M., Peterson, A.: Dynamic Equations on Time Scales, an Introduction with Applications. Boston, Birkhauser (2001)

7. Saker, S.H.: Oscillation of second-order nonlinear neutral delay dynamic equations on time scales. J. Comput. Appl. Math. 187, 123-141 (2006)

8. Han, Z., Li, T., Sun, S.: Oscillation for second-order nonlinear delay dynamic equations on time scales. Adv. Differ. Equ. 2009, 756171 (2009)

9. Sun, Y., Han, Z., Sun, S.: Oscillation of a class of second order half-linear neutral delay dynamic equations with damping on time scales. Acta Math. Appl. Sin. 36, 480-494 (2013)

10. Agarwal, R.P., Bohner, M., Li, T.: Oscillation criteria for second-order dynamic equations on time scales. Appl. Math. Lett. 31, 34-40 (2014)

11. Han, Z., Li, T., Sun, S.: Remarks on the paper [Appl. Math. Comput. 207 (2009) 388-396]. Appl. Math. Comput. 215 3998-4007 (2010) 
12. Yang, J., Tan, W., Qin, X.: Oscillation analysis of certain second-order nonlinear damped dynamic equations on time scales. J. Zhejiang Univ. Sci. Ed. 43, 64-70 (2016)

13. Deng, X., Wang, Q., Zhou, Z.: Oscillation criteria for second order neutral dynamic equations of Emden-Fowler type with positive and negative coefficients on time scales. Sci. China Math. 60, 113-132 (2017)

14. Bohner, M., Li, X.: Oscillation of second-order $p$-Laplace dynamic equations with a nonpositive neutral coefficient. Appl. Math. Lett. 37, 72-76 (2014)

15. Zhang, C., Agarwal, R.P., Bohner, M.: New oscillation results for second-order neutral delay dynamic equations. Adv. Differ. Equ. 2012, 227 (2012)

16. Yang, J., Qin, X.: Oscillation criteria for certain second-order Emden-Fowler delay functional dynamic equations with damping on time scales. Adv. Differ. Equ. 2015, 97 (2015)

17. Tang, S., Gao, C., Li, T.: Oscillation theorems for second-order quasi-linear delay dynamic equations. Bull. Malays. Math. Sci. Soc. 36, 907-916 (2013)

18. Yang, J., Fang, B.: Oscillation analysis of certain second-order nonlinear delay damped dynamic equations on time scales. Math. Appl. 30, 16-26 (2017)

19. Yang, J.: Oscillation criteria for certain third-order delay dynamic equations. Adv. Differ. Equ. 2013, 1 (2013)

20. Yang, J.: Oscillation for a class of second-order Emden-Fowler dynamic equations on time scales. Acta Math. Appl. Sin. 39, 334-350 (2016)

21. Li, T., Saker, S.H.: A note on oscillation criteria for second-order neutral dynamic equations on isolated time scales. Commun. Nonlinear Sci. Numer. Simul. 19, 4185-4188 (2014)

22. Deng, X., Wang, Q., Zhou, Z:: Oscillation criteria for second order neutral dynamic equations of Emden-Fowler type with positive and negative coefficients on time scales. Sci. China Math. 60, 113-132 (2017)

23. Qiu, Y., Wang, H., Jiang, C., Li, T.: Existence of nonoscillatory solutions to third-order neutral functional dynamic equations on time scales. J. Nonlinear Sci. Appl. 11, 274-287 (2018)

\section{Submit your manuscript to a SpringerOpen ${ }^{\circ}$ journal and benefit from:}

- Convenient online submission

- Rigorous peer review

- Open access: articles freely available online

- High visibility within the field

Retaining the copyright to your article

Submit your next manuscript at $\gg$ springeropen.com 